\title{
Practical Design and Flight Test of a Yo-Yo Wire-Boom Deployment System
}

\author{
Mark L. Psiaki, ${ }^{*}$ Steven P. Powell, ${ }^{\dagger}$ Eric M. Klatt, ${ }^{\ddagger}$ and Paul M. Kintner Jr. ${ }^{\S}$ \\ Cornell University, Ithaca, New York 14853-7501
}

\begin{abstract}
A yo-yo-type wire-boom deployment system has been developed and flight tested on a sounding rocket mission. The goal of the work has been to validate a new mechanism that rapidly deploys wire booms from a spinning spacecraft. This work takes a theoretical system design and implements it in practical hardware. The limitations inherent in practical hardware necessitated new theoretical developments. A modified stability analysis has been developed for the case of nonzero axial separation between the wire-boom base attachment points and the spacecraft's center of mass. This modified stability analysis dictates that a stable design is impractical for many missions because very large wire-boom tip masses are needed and because the three-dimensional deployment transients are very sensitive to small asymmetries. This problem has led to the development of design criteria and analysis techniques, which permit a short-duration mission to use a slightly unstable nutation mode. These techniques have been used to design a system that has been flown on two daughter spacecraft that were part of a formation of three sounding rocket subpayloads. Each daughter spacecraft deployed four 3-m-long wire booms in under $10 \mathrm{~s}$ and maintained a low level of spin instability for the remaining $700 \mathrm{~s}$ of the mission. The nutation oscillations showed a slow exponential growth, but the coning half-angles of both spacecraft never exceeded $16 \mathrm{deg}$.
\end{abstract}

\section{Introduction}

$\mathbf{T}$ HE present paper deals with the problem of how to use wireboom systems on a short-duration space flight such as a sounding rocket mission. Wire booms can be deployed from a spinning spacecraft and are held in their extended positions by centrifugal force. They can be used to create large, ultralightweight antenna or probe structures. Wire booms have been used on several spacecraft missions in the past. ${ }^{1,2}$ Wire-boom structures offer an attractive replacement for the telescoping boom systems that are typically used on sounding rocket missions because they can be built with greater length while using less mass. The main obstacle to using wire-boom systems on sounding rockets has been their long deployment times. The systems described in Refs. 1 and 2 deployed their booms over a span of hours or days, but a sounding rocket flight lasts only 1000$2000 \mathrm{~s}$.

A recent simulation study proposed a new wire-boom deployment system that is practical for sounding rockets because it deploys in a matter of seconds. ${ }^{3}$ This system is an adaptation of the classic yoyo despin device that is described in Refs. 4 and 5. The adaptation provides a means of retaining the yo-yos as wire booms without their rewrapping about the spacecraft. Rewrapping of yo-yos results when both energy and angular momentum are conserved.

The system described in Ref. 3 incorporates an energy dissipation mechanism that prevents rewrapping. It consists of a cylindrical drum that can rotate with respect to the main spacecraft body under the influence of a rotary damper. The boom wires are wrapped about this drum and deploy by unwrapping from it. The drum's rotary articulation axis is aligned with the spacecraft's nominal spin axis, and the drum and the spacecraft are initially constrained to spin together. The deployment starts when a wire cutter simultaneously

Received 29 April 2003; revision received 28 May 2004; accepted for publication 23 June 2004. Copyright (C) 2004 by the authors. Published by the American Institute of Aeronautics and Astronautics, Inc., with permission. Copies of this paper may be made for personal or internal use, on condition that the copier pay the $\$ 10.00$ per-copy fee to the Copyright Clearance Center, Inc., 222 Rosewood Drive, Danvers, MA 01923; include the code 0731-5090/05 \$10.00 in correspondence with the CCC.

*Associate Professor, Sibley School of Mechanical and Aerospace Engineering. Associate Fellow AIAA.

${ }^{\dagger}$ Research Support Specialist, School of Electrical and Computer Engineering.

* Graduate Student, School of Electrical and Computer Engineering.

${ }^{\S}$ Professor, School of Electrical and Computer Engineering. releases the weighted tips of the wire booms and the drum's rotational degree of freedom. The tension that builds up in the wire booms during the deployment slows the drum down so that it spins with respect to the main spacecraft body. This relative motion is then damped by the rotary damper. If the damping law has been designed to dissipate energy properly, then the wire booms deploy to their fully extended positions in $10-20 \mathrm{~s}$, and they remain there with slight oscillations, but without significant rewrapping.

Reference 3 develops an analytical model of the yo-yo wire-boom deployment system, derives a global stability criterion, and performs simulation studies. Its model is nonlinear and includes 12 degrees of freedom. It is valid during the initial transient unwrapping motion of the wires and during the steady-state oscillatory motion that occurs after deployment. Reference 3 demonstrates that the system can be designed to deploy robustly in the face of typical levels of manufacturing error and initial condition asymmetry.

The present paper extends the analyses of Ref. 3 to allow designs that are more practical, and it reports on ground-test and flight experience with an actual system. This system flew aboard the sounding rocket mission known as SIERRA (sounding of the ion energization region: resolving ambiguities). One new analysis extends the stability criterion to the case where the wire-boom attachment points are axially displaced from the system center of mass. These results and related simulation studies led to the conclusion that a stable design was unworkable for the SIERRA mission. Therefore, additional analysis capabilities and design criteria have been developed to allow slightly unstable designs. Another section of the present paper deals with the selection and evaluation of a practical semiactive damper for the energy dissipation system. This paper's final contributions are to report the results from ground tests and from the first flight of this new system. Data from these experiments are used to evaluate the new system's efficacy and to assess the accuracy of simulation-based performance predictions.

The remainder of this paper presents new analyses, simulation results, and experimental results in six sections and an appendix. Section II and the appendix develop a modified stability criterion for the system's nutational motion. Section III explains the design considerations that led to the acceptance of an unstable design for the SIERRA mission. Section IV presents new analysis techniques and design criteria that accommodate the use of a slightly unstable nutation mode. Section $\mathrm{V}$ describes the semi-active damper/brake that is part of the SIERRA system, and it presents an experimentally determined brake model that has been used in SIERRA system studies. Section VI gives the results of ground tests of the 
deployment system, and Sec. VII presents flight results from the SIERRA mission. The paper's conclusions are summarized in Sec. VIII.

\section{Stability When the Boom Attachment Point Has an Axial Offset from the Center of Mass}

A designer might need to constrain the system's steady-state deployed configuration to be passively stable. The deployed configuration of the SIERRA system is depicted on the right-hand side of Fig. 1. The drum about which the booms were wrapped when they were stowed has a radius of $\rho_{r}$, the booms have a length of $l_{f}$, and each boom is modeled as being massless except for a concentrated tip mass equal to $m_{\text {tip. }}$. Distributed cable mass is included by lumping $\frac{1}{3}$ of it into $m_{\text {tip }}$. This approach is a reasonable simplification for the light wire booms that have been used $;^{3}$ their linear densities are on the order of $8 \mathrm{gr} / \mathrm{m}$. The SIERRA system uses four wire booms that are mounted 90 deg apart from each other around the drum. The center of mass depicted in Fig. 1 is for the entire system excluding the boom tip masses. This center of mass has a $z$-axis displacement from the base attachment points of the booms. This displacement is called an axial displacement because it is measured along the nominal spin axis. Its value is $z_{r}$. In the stowed configuration, the axial displacement of the wire-boom tip masses from the booms' base attachment points is $z_{0}$. When fully deployed, the position of the $i$ th wire boom with respect to the drum is characterized by its in-plane pendulum angle $\theta_{i}\left(+\theta_{i}\right.$ rotation occurs about the $-z$ axis) and its out-of-plane pendulum angle $\phi_{i}$ (positive rotation moves its tip mass in the $+z$ direction).

The system is stable if the spacecraft spin direction tends to realign itself with the body $z$ axis after it has been perturbed, but this perturbation will grow for an unstable configuration. If the system is unstable, then the spin vector's growing oscillations are very similar to the unstable nutations that occur when a minor-axis spin-stabilized rigid spacecraft experiences nutation-induced energy dissipation. The stability of the nominal configuration can be determined by consideration of the system's kinetic energy for a fixed angular momentum magnitude. The system is stable if and only if the kinetic energy is a minimum when the spacecraft is spinning in its nominal configuration. The following inequality provides a design criterion to ensure that the nominal spin axis is at least a local minimum energy state:

$$
I_{\text {zzbd }}+2 m_{\text {tip }} \rho_{r}\left(\rho_{r}+l_{f}\right)>I_{\text {xxbd }}+z_{r}^{2}\left[\frac{4 m_{\text {tip }}\left(1+l_{f} / 2 \rho_{r}\right) m_{\text {bd }}}{4 m_{\text {tip }}\left(1+l_{f} / 2 \rho_{r}\right)+m_{\text {bd }}}\right]
$$

where the moment-of-inertia matrix of the combined mainspacecraft-body/drum system about its center of mass is $\operatorname{diag}\left(I_{\mathrm{xxbd}}, I_{\mathrm{xxbd}}, I_{\mathrm{zzbd}}\right)$ in $x-y-z$ body coordinates and where $m_{\mathrm{bd}}$ is the mass of the main-body/drum system. Note that the subscript ${ }_{b d}$ is used throughout the paper to refer to the main-spacecraft-body/drum system. A derivation of Eq. (1) is contained in the Appendix. Equation (1) is a generalization to the case of four wire booms of Ref. 6's Eq. (33); the condition in Ref. 6 is for the stability of a spinning spacecraft that has only two wire booms.

The Eq. (1) stability criterion is an extension of the criterion given in Eq. (13) of Ref. 3. The extension deals with the case of a nonzero axial offset between the main-payload/drum center of mass and the wire-boom base attachment points $z_{r}$. This extension has been necessitated by practical design considerations. It is obvious from Eq. (1) that a nonzero $z_{r}$ makes the system less stable, which is why the initial design enforced $z_{r}=0$. It proved impractical to create a drum/bearing/damper system that could achieve $z_{r}=0$ for the mass and envelop constraints of the SIERRA mission. A design that has a drum mounted on one end, as shown in Fig. 1, is much more practical. In this case, a single semi-active brake can provide both damping and the bearing system that allows drum articulation. This brake is mounted coaxially with the drum just under the drum's top surface as seen in Fig. 1.

The stability criterion in Eq. (1) is more restrictive than it would be if the booms were rigid. Stability would be guaranteed for rigid booms if the spacecraft were a major-axis spinner because spin about the major inertia axis yields the minimum energy for a fixed angular momentum. In this case, the stability criterion is

$$
I_{\mathrm{zzbd}}+2 m_{\mathrm{tip}}\left(\rho_{r}+l_{f}\right)^{2}>I_{\mathrm{xxbd}}+z_{r}^{2}\left(\frac{4 m_{\mathrm{tip}} m_{\mathrm{bd}}}{4 m_{\mathrm{tip}}+m_{\mathrm{bd}}}\right)
$$

for a four-boom system. This criterion is less restrictive than Eq. (1) for two reasons: The second term on the left-hand side is always larger than the corresponding term on the left-hand side of Eq. (1),

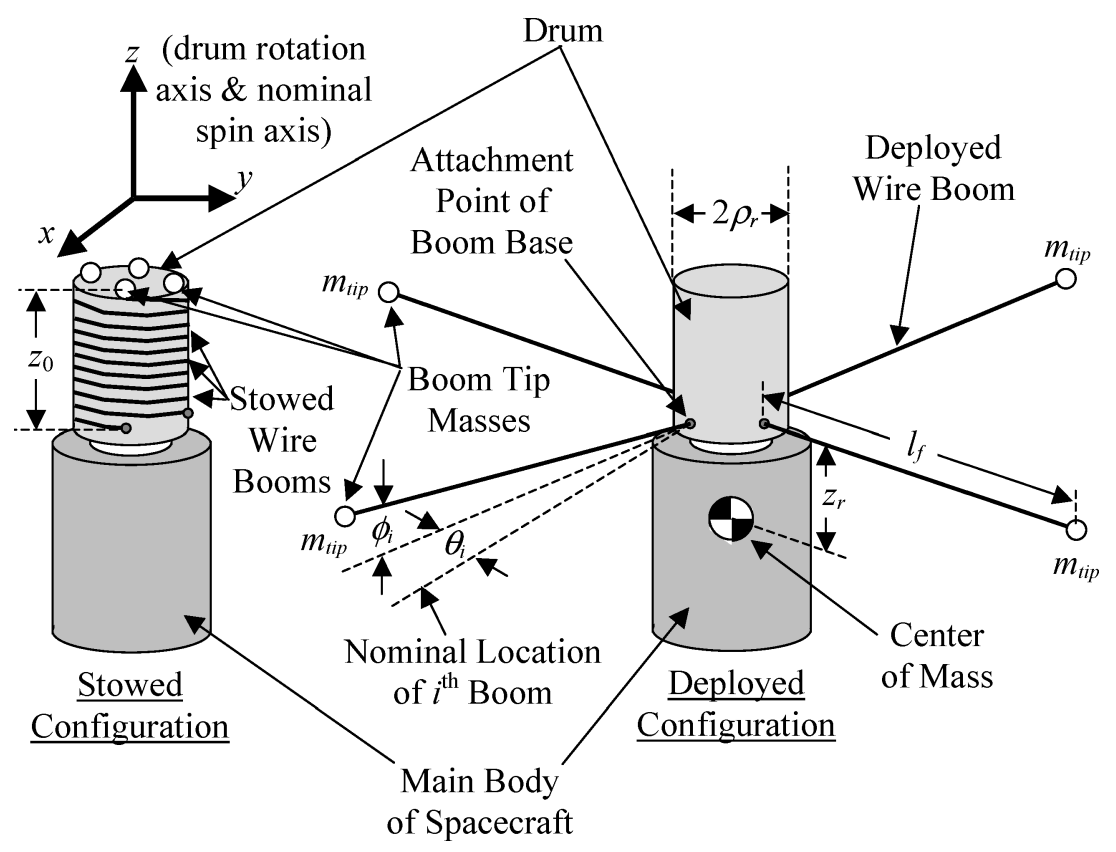

Fig. 1 Stowed and deployed configurations of the SIERRA wire-boom system. 
and the second term on the right-hand side is always smaller than the corresponding term on the right-hand side of Eq. (1). Thus, the domain of the design parameter space that stabilizes a wire-boom system is more restricted than the domain that stabilizes an equivalent system with rigid booms that have equivalent mass properties.

A stable SIERRA design was developed by varying the boom tip mass and length parameters $m_{\text {tip }}$ and $l_{f}$, while holding other major design parameters fixed at the values

$$
\begin{gathered}
m_{\mathrm{bd}}=45.43 \mathrm{~kg} \\
I_{\mathrm{xxbd}}=1.741 \mathrm{~kg}-\mathrm{m}^{2}, \quad I_{\mathrm{zzbd}}=0.807 \mathrm{~kg}-\mathrm{m}^{2} \\
z_{r}=0.0664 \mathrm{~m}, \quad \rho_{r}=0.152 \mathrm{~m}
\end{gathered}
$$

This is a reasonable design strategy because large variations in $m_{\text {tip }}$ and $l_{f}$ have no major impact on other parts of the design. Furthermore, the stability criterion in Eq. (1) can be satisfied if $m_{\text {tip }}, l_{f}$, or both are set to large enough values. The following candidate values were chosen because they provide a reasonable degree of stability:

$$
m_{\text {tip }}=0.726 \mathrm{~kg}, \quad l_{f}=5.567 \mathrm{~m}
$$

Using these parameter values, the left-hand side of Eq. (1) equals $2.069 \mathrm{~kg}-\mathrm{m}^{2}$, and the right-hand side is $1.852 \mathrm{~kg}-\mathrm{m}^{2}$; so, the nutation mode of the system is stable. The tip-mass value $0.726 \mathrm{~kg}$ seems large, but Eq. (1) dictates the lower bound $m_{\text {tip }}>0.595 \mathrm{~kg}$ for stability at the given $l_{f}$ value. One might have tried to increase $l_{f}$ in order to allow $m_{\text {tip }}$ to decrease, but this seemed unreasonable because $l_{f}$ was already long enough to raise concerns that the wire booms might tangle during the deployment if tip-off or manufacturing errors caused the system to start with a small initial nutational wobble.

\section{Reasons to Relax the Nutational Stability Requirement for the SIERRA Mission}

The stable design of Sec. II can experience problems during deployment. The possibility of problems was revealed by a simulation study of the deployment transients. The study considered both nominal and perturbed situations. Perturbations included small asymmetries in the spacecraft caused by manufacturing errors and small initial condition asymmetries. The levels of these asymmetries were sized to correspond to expected values based on design tolerances and prior flight experience with similar systems. Reference 3 contains additional discussion of simulation-based studies of the effects on this system of parametric and initial condition uncertainties.

The stable design allows a small initial nutational wobble to build up during the transient part of the deployment. This asymmetric motion can cause the four wire booms to unwrap at significantly different rates. Nominally, the wire booms remain 90 deg away from each other as measured around the circumference of the wirewrapping drum. During asymmetric deployment, however, one wire boom can unwrap much faster than its neighbors, and it can pass the neighboring wire boom that is supposed to remain ahead of it. The deployment design criteria stipulate that this must never happen because such a situation could lead to entanglement of the wire booms. In this case, the satisfaction of a global minimumenergy stability criterion would be of little value because the initial entanglement could cause the system to become entrapped in a local minimum-energy condition in which the wires remained entangled forever.

Although Ref. 3 considered the fast, nonlinear, three-dimensional deployment transients of these wire boom systems in order to determine whether they could result in a flat spin or wire entanglement, it failed to predict the present design's entanglement problem for two reasons. First, Ref. 3 only considered cases whose nominal axial displacement between the main-body center of mass and the wireboom base attachment points was $z_{r}=0$. Second, Ref. 3 presumed that the wires would be wrapped on alternate sides of their base attachment points. In other words, it presumed that the $z_{0}$ initial axial locations of neighboring tip masses would have opposite signs (review Fig. 1). Such designs have a balance that tends to minimize the buildup of wobble during the deployment. The SIERRA design depicted in Fig. 1 has a significant positive $z_{r}$, and $z_{0}$ is large and positive for all of the booms. The nominal $z_{0}=0.304 \mathrm{~m}$. These two facts, when coupled with the large tip-mass values, cause any initial wobble to grow during the deployment transients. The large masses cause large transient tensions in the wires. Asymmetries allow these tensions to impart large transverse torques to the main spacecraft body. These torques act to increase the wobble, which increases the asymmetries, which increase the transverse torques, and the wobble grows to the point where the wire booms can become entangled.

A number of different designs have been tried in order to alleviate this problem. Given the design constraints of significant positive $z_{r}$ and $z_{0}$ values, the only designs that yield acceptable deployment transients are ones that use very small tip-mass values, that is, $m_{\text {tip }}$ on the order of $0.090 \mathrm{~kg}$ or less. Such values produce robust deployment transients. The expected levels of asymmetry do not produce rapid growth of transient wobble motion when $m_{\text {tip }}$ is small, which eliminates large variations in wire unwrapping rates and the possibility that wire booms will become entangled during the deployment. Unfortunately, small values of $m_{\text {tip }}$ give the SIERRA design an unstable nutation mode because they violate the stability criterion in Eq. (1).

\section{Design and Analysis of a Slightly Unstable Nutation Mode}

The superior deployment robustness of designs that use small tipmass values led to the question of whether an acceptable unstable design could be found for the SIERRA mission. Such a suggestion would be out of the question for a long orbital mission, but a sounding rocket mission can accept a slightly unstable attitude dynamics mode because of the limited duration of its flight. In fact, it is common practice to use minor-axis spin stabilization for sounding rockets even when they contain long semirigid booms whose energy dissipation destabilizes the nutation mode.?

\section{A. Design Criteria for the Unstable Nutation Mode}

The design strategy that was adopted for SIERRA was to allow the nutation mode to be slightly unstable if the resultant coning motion could be guaranteed to respect prescribed upper bounds on the size of the coning half-angle that would occur at various key phases of the mission. Such bounds translate into a combined limit on the rate of growth of the unstable mode and on the worst-case initial coning amplitude. The rate of growth of the coning mode can be controlled by minimizing the amount of damping in the wire booms as they undergo pendulum-type motion. In particular, energy dissipation during out-of-plane $\phi_{i}$ pendulum angle motion must be kept small (review Fig. 1). The worst-case initial coning half-angle is dependent on three factors: 1) manufacturing tolerances, 2) the accuracy of the feedback-controlled spin motion at the time when each wire-boom-configured SIERRA daughter spacecraft separates from the mother spacecraft, and 3) the possible magnitude of the lateral tip-off torque during separation.

The growth rate of the unstable nutation mode depends primarily on the damping rate of the out-of-plane pendulum motion of the wire booms. This damping rate is parameterized by the damping constant $b_{c}$ in the analysis of Ref. 3 . The main source of energy dissipation in a wire boom is structural damping of the wire's bending deformation as the tip mass undergoes pendulum motion. This bending occurs primarily at the boom base. Damping can be minimized by choosing a wire material that has low damping, by developing an attachment design for the wire base that minimizes any possible relative motion between parts such as slipping or rubbing, or by modifying the wire near the base attachment point in order to reduce its structural damping during bending. One such modification that has been tried for another project is to strip off an outer insulation cover and a Kevlar layer from $4 \mathrm{~cm}$ of the wire at the base of the boom. This small modification almost halves the net damping during pendulum motion. The wire that has been chosen for the current mission is a coaxial wire with a Teflon ${ }^{\circledR}$ outer insulation layer. Its outer diameter is $0.00178 \mathrm{~m}(0.070 \mathrm{in}$.). 
The initial coning half-angle has been limited by enforcing tight requirements on the initial spin direction, on the orientation of the spacecraft principal spin-axis direction, and on the possible lateral tip-off torques during separation of the daughter spacecraft from the mother vehicle. The spin direction has been controlled through requirements on the mother spacecraft's attitude control system. The principal axis has been controlled by a preflight spin-balancing procedure that has used a spin table to make inertia measurements. The lateral tip-off has been bounded by requirements on the uniformity of the separation springs' behavior and by distributing the separation impulse over a number of springs. The final design has a worst-case initial coning half-angle of $3 \mathrm{deg}$.

\section{B. Analysis of Worst-Case Coning Growth}

The worst-case expected coning angle growth can be determined via simulation. The simulation used in this study is based on the three-dimensional model given in Ref. 3, but with one difference. The model has been augmented to include the effect of a locking brake that acts between the main spacecraft body and the wirewrapping drum. This brake locks out relative rotation after the transient part of the deployment has finished and after the energy dissipation that keeps the wires from rewrapping has been accomplished. It is activated by a timer. Once activated, this brake freezes the drum articulation angle $\theta_{r}$ at a constant value. There are two reasons for locking out the drum rotation at the end of the initial deployment. One is to reduce energy losses that have the potential to lead to nutation growth. The other is to allow the semiactive damper coils to be turned off so that they cannot contaminate the science magnetometer data. The simulation models the activation of the brake as an inelastic collision followed by a period in which $\dot{\theta}_{r}$ is constrained to be zero.

The accuracy of the prediction of the growth in SIERRA's unstable nutation mode is completely dependent on the accuracy of the wire damping rate parameter $b_{c}$. Therefore, a series of laboratory experiments have been performed to get an estimate of $b_{c}$. These experiments have used the flight wire to create a simple pendulum in a $1-g$ environment whose decay rate has been measured as a function of the wire's length and nominal tension. The test apparatus has included pendulum base attachment hardware that is the same as that used on the SIERRA mission at the base attachment points of the wire booms. The amplitude time history was recorded using a simple engineering scale to measure amplitude, a clock to measure time, and a paper and a pencil to record data. This data recording scheme was acceptable because the pendulum oscillation periods used in the tests were relatively long, on the order of 1.4-3.5 s. The pendulum decay rate measurements have been analyzed using physics and mathematics in order to estimate the correct value of $b_{c}$ to use in the nonlinear simulation of SIERRA's flight performance.

Structural damping produces an energy loss per oscillation cycle that is approximately proportional to the square of the small zeroto-peak deformations of the structure and that is independent of frequency. This loss is typically modeled by a force or torque that is linearly proportional to displacement but 90 deg out of phase with displacement. ${ }^{8}$ Suppose that the structural loss per pendulum cycle is $K_{\text {loss }} \phi_{0-\text { pk }}^{2}$, where $K_{\text {loss }}$ is a constant that is dependent on the wire boom material, the attachment geometry, and the nominal wire tension and where $\phi_{0-\mathrm{pk}}$ is the equivalent instantaneous zeroto-peak displacement of the pendulum. The energy loss constant $K_{\text {loss }}$ can be used to predict the decay rate of the pendulum during an experiment, and it can be used to determine $b_{c}$ for use in the SIERRA flight simulation. If one makes the reasonable assumption that the energy loss per cycle is a small fraction of the total energy, then the time history of the zero-to-peak pendulum angle during each experiment takes the form

$$
\begin{gathered}
\phi_{0-\mathrm{pk}}(t)=\phi_{0-\mathrm{pk}}(0) e^{-a t} \quad \text { with decay coefficient } \\
a=\frac{K_{\mathrm{loss}} \omega}{2 \pi m g l}=\frac{K_{\text {loss }}}{2 \pi m \sqrt{g l^{3}}}
\end{gathered}
$$

where $\omega$ is the pendulum oscillation frequency, $m$ is its tip mass, and $l$ is its length. The following cable damping coefficient gives an

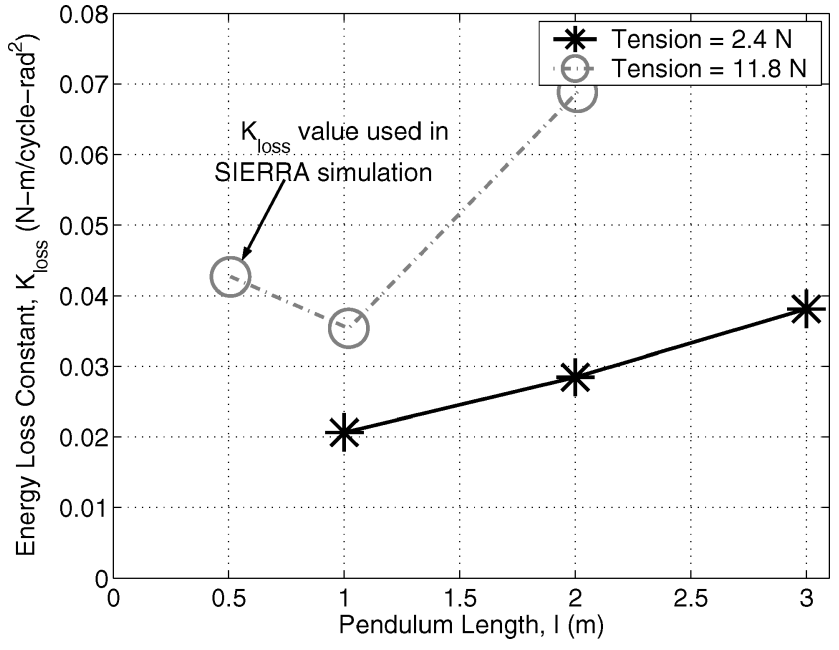

Fig. 2 Wire damping loss constant as a function of pendulum length and tension.

equivalent loss per cycle per radian squared of zero-to-peak deflection when used in the simulation of the wire-boom system:

$$
b_{c}=\frac{K_{\mathrm{loss}}}{\pi \omega_{\mathrm{nut}} l_{f}^{2}}
$$

where $\omega_{n u t}$ is the nutation-mode oscillation frequency and $l_{f}$ is the length of each wire boom.

The damping constant $b_{c}$ can be determined by using a pendulum experiment. Given $a$ from an appropriate experiment, $K_{\text {loss }}$ can be deduced from Eq. (3), and $K_{\text {loss }}$ can be used in Eq. (4) to determine $b_{c}$. One must be careful, however, about two effects. Aerodynamic losses occur in the pendulum experiment, but not in flight, and the parameter $K_{\text {loss }}$ depends on the nominal wire tension.

The effects of tension and aerodynamics can be accounted for properly through careful design of the pendulum experiment. One deals with the tension dependence simply by choosing the pendulum tip mass so that the nominal tension during the pendulum test $m g$ equals the nominal flight tension during the mission. An analysis has been made of the aerodynamic drag losses in the cable and the tip mass. The aerodynamic loss per cycle is a decreasing function of the wire-boom length used in the pendulum experiment. Thus, one should determine $K_{\text {loss }}$ based on a pendulum test that uses a sufficiently short length.

The effects of tension and of aerodynamics are illustrated by Fig. 2, which shows experimentally determined plots of $K_{\text {loss }}$ as a function of $l$ for two different nominal cable tensions. The nominal SIERRA cable tension is $11.8 \mathrm{~N}$, which corresponds to the gray dash-dotted curve with the o symbols. The solid black curve with the $*$ symbols corresponds to a tension that equals $\frac{1}{5}$ of the nominal SIERRA tension. The markedly different levels of these two curves prove that the structural losses are tension dependent. This conclusion is altogether plausible when one considers that the losses are a function of the wires' bending stress-strain relationship, which can vary as a function of the nominal load. These two curves also illustrate that the loss per cycle per radian ${ }^{2}$ decreases as length decreases, as predicted by aerodynamic analysis. This analysis concludes that the aerodynamic losses are negligible for $l=1 \mathrm{~m}$ for the SIERRA wire, which implies that the curves should flatten out in this $l$ range. The gray curve not only flattens out, but it actually increases as $l$ decreases from 1 to $0.5 \mathrm{~m}$. This increase is probably the result of experimental error. The black curve does not include enough data to show the flattening effect at low $l$ values, but another set of tests that used a wire for another mission and the same tension as the lower curve showed a clear flattening below $l=1 \mathrm{~m}$.

The $a$ decay coefficient values that have been used to determine the $K_{\text {loss }}$ values of Fig. 2 have been estimated based on the initial few minutes of data from each pendulum test. These tests started with 
zero-to-peak pendulum angles ranging from $6 \mathrm{deg}$ for the $l=0.5$ and $1-\mathrm{m}$ tests down to $2 \mathrm{deg}$ for the $l=3-\mathrm{m}$ tests. Exponential decay of the zero-to-peak amplitude provides a good model of the dynamics during the initial transients, but later transients show increased decay, probably caused in part by coupling into the initial unperturbed direction of oscillation and in part by a nonlinear effect whereby $K_{\text {loss }}$ increases as the zero-to-peak amplitude decreases. The possibility of this latter nonlinear effect makes it all the more important to use the initial data at larger amplitudes in order to estimate $K_{\text {loss. }}$. Otherwise, the loss predictions will be too conservative because small amplitudes, and their corresponding larger $K_{\text {loss }}$ values, will not apply for much of a flight because energy loss is what drives increases in the nutation amplitude.

The pendulum analysis results have been used to verify that the final SIERRA design meets the limit on coning half-angle. This design uses the wire boom parameters $m_{\text {tip }}=0.0343 \mathrm{~kg}$ and $l_{f}=$ $2.837 \mathrm{~m}$. The resulting nutation frequency is $\omega_{\text {nut }}=5.07 \mathrm{rad} / \mathrm{s}$. The $K_{\text {loss }}$ value from Fig. 2 for the 0.5 -m pendulum test with the nominal SIERRA tension has been used in Eq. (4) to compute the cable damping coefficient $b_{c}=3.33 \times 10^{-4} \mathrm{~N}-\mathrm{s} / \mathrm{m}$. One might argue that the higher $K_{\text {loss }}$ value at $l=0.5 \mathrm{~m}$ in Fig. 2 is too conservative and that one should use the average of the $K_{\text {loss }}$ values at $l=0.5$ and $1.0 \mathrm{~m}$ in order to reduce the impact on the flight simulation of experimental errors during the pendulum tests. The higher of these two values has been used in order to be conservative.

Figure 3 depicts the coning half-angle time history of a typical simulation for the final SIERRA design. The coning half-angle is the angle between the nominal spin axis and the total system angular momentum vector. Asymmetric initial conditions cause the coning half-angle to be about 4 deg after the initial deployment, and the damping coefficient from Eq. (4) causes the coning half-angle to grow to $20 \mathrm{deg}$ by $700 \mathrm{~s}$ after the deployment, which is the nominal end time of the mission. Note that time equals zero when the wire booms begin to deploy. The mission requirement was for the coning half-angle to remain below $45 \mathrm{deg}$ for the duration of the mission. Thus, the design meets the mission requirement.

This method of using pendulum tests to determine $b_{c}$ and the rate of coning growth is somewhat different from what was actually used before the SIERRA launch. The original preflight pendulum test did not use flight hardware at the pendulum root, it did not use the correct $m$ to get the correct wire tension, and it did not use a precise aerodynamic damping analysis along with tests at different wire lengths in order to derive a $b_{c}$ value that was not biased by aerodynamic effects. Analysis of the preflight data also failed to properly account for the flight nutation frequency in the computation of $b_{c}$, as in Eq. (4). All of these shortcomings combined to cause it to predict a much larger rate of coning growth, to about 50 deg of coning half-angle $700 \mathrm{~s}$ after deployment. The new experimental/analysis

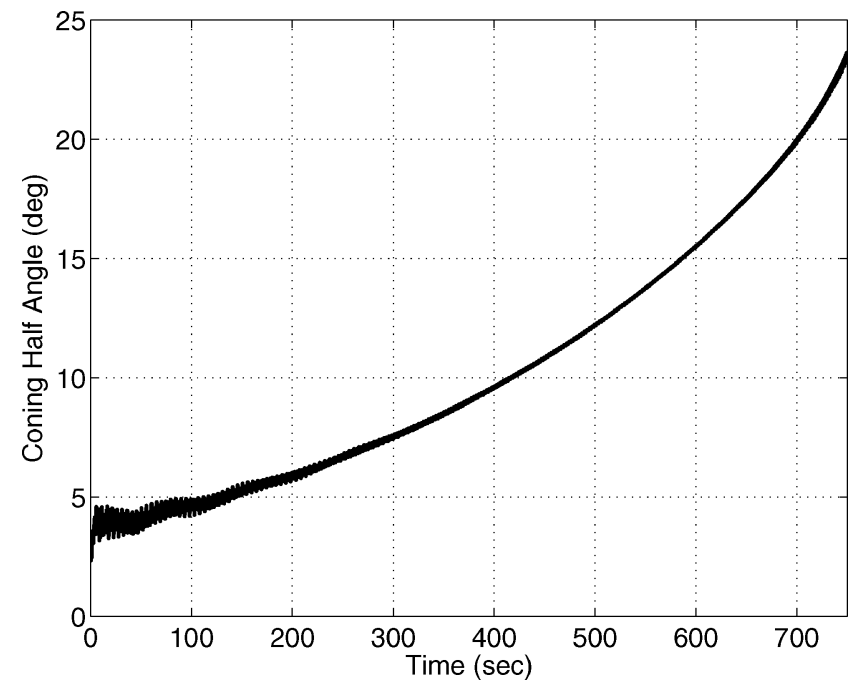

Fig. 3 Simulated coning half-angle time history for the slightly unstable SIERRA design. technique has been developed in support of a new mission, which uses different wire and longer wire-boom lengths. The process of doing experiments to determine the new $b_{c}$ value uncovered the problems that were inherent in the method that was used to make preflight predictions of SIERRA's rate of coning growth.

One might question the validity of using pendulum tests in a 1-g environment to predict damping in a $0-g$ environment. All of the analysis and test data agree with the following three points: gravity, per se, is not a direct factor in determining damping in either the pendulum tests or in the flight performance, structural damping is an important factor in both situations, and aerodynamics can be significant for ground-based pendulum tests with long pendulum lengths. Gravity affects the loss in the pendulum tests only in that it affects the nominal tension in the cable, which affects the amount of structural damping. If one sizes the pendulum tip mass for the tests properly, that is, to simulate the nominal spin-induced tension during flight, then the structural loss per cycle during the pendulum tests will be equivalent to that experienced during flight.

Another question that naturally arises is that of energy loss caused by longitudinal deflections of the wire booms. During nutational oscillations, the wire-boom tensions vary with time, and these variations will give rise to slight length changes caused by the wires' finite stiffness. Given that the beam's longitudinal flexure modes are more than two orders of magnitude faster than the nutation mode, the maximum possible energy loss per cycle is $\pi \Delta T^{2} / k$ if one assumes viscous longitudinal damping. The quantity $\Delta T$ is the zero-to-peak tension variation during nutation, and the quantity $k$ is the wireboom's longitudinal stiffness. If one assumes Coulomb damping, then the maximum energy loss per cycle is reduced by a factor of $\pi$. The measured longitudinal stiffness of the SIERRA wire booms is $k=7700 \mathrm{~N} / \mathrm{m}$, and the nutation-induced tension variations are $\Delta T \cong 0.3 \mathrm{~N}$ when the coning half-angle is $10 \mathrm{deg}$. Thus, the maximum possible longitudinal energy loss summed over the four wire booms is $1.5 \times 10^{-4} \mathrm{~N}-\mathrm{m} /$ cycle. The energy loss rate that explains the rate of coning half-angle growth, however, is $1.0 \times 10^{-2} \mathrm{~N}$ $\mathrm{m} /$ cycle. Therefore, it is impossible to explain a significant amount of the SIERRA coning half-angle growth as being the result of structural damping in the longitudinal deflections of the wire booms. The boom lengths are too stiff to allow significant energy loss by this mechanism.

\section{Design and Analysis of the Semiactive Braking System}

One of the major design challenges that Ref. 3 left unsolved concerned how to design a practical damper that would dissipate the excess energy of the yo-yo deployment. Reference 3 found that the damping torque has to be a nonlinear function of the relative angular velocity between the drum and the main spacecraft body. The damping law has a higher damping rate at high rotation rates and a lower damping rate at low rotation rates. This type of damping law yields a robust deployment that keeps the wires unwrapping at a relatively constant rate while avoiding large in-plane $\theta_{i}$ pendulum motion after the wires are fully unwrapped.

The practical design problem concerns how to achieve the necessary damping law using a lightweight design. A passive viscous damper cannot be designed to obey the required law. The damping law can be implemented using a dc-motor and active feedback, but the mass of such a system is much too large, and the transient power usage is also on the high side.

A practical solution to this design problem is to use a semi-active brake. It feeds the sensed relative angular velocity between the main spacecraft body and the drum back to a magnetorheological brake. The brake contains a viscous oil that has iron filings in it, and it includes electromagnetic coils. If current is passed through the coils, then the iron filings tend to line up, and this tendency increases the viscosity of the oil/filings mixture. The result is a lightweight brake whose braking torque can be controlled by the current in the coil. The braking torque is independent of speed. The brake that has been used is the aerobic exerciser brake that is described in Ref. 9.

Although the brake manufacturer publishes a model for the brake's performance, extensive bench tests have been performed 
Temporary Fly-Away

Drive Belt Connection to Spin-Up Motor

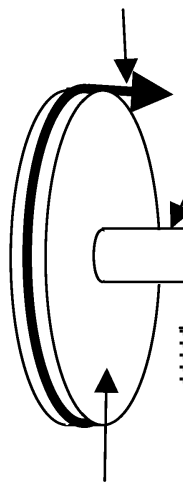

Rotor

Inertia

\section{Brake Shaft}

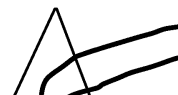
Input Lines
Power

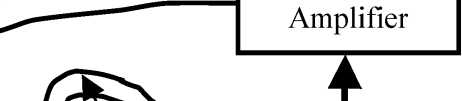

Rheological

Brake

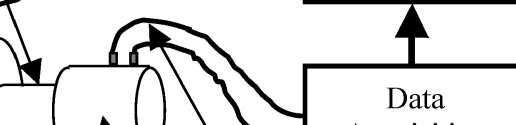

Data

and Control

Computer

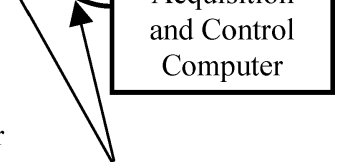

Tachometer

Voltage Output Lines

Fig. 4 Diagram of test apparatus for measuring the magnetorheological brake's torque/current/speed relationships.

on two individual brakes in order to develop an accurate model. This model has been needed in order to accurately simulate the brake's effect on the deployment dynamics of the wire-boom system. The experimental setup for these tests is depicted in Fig. 4. The brake has been connected to a rotor with a significant known moment of inertia that is much larger than the moment of inertia of the brake's rotor, and each bench test has started with the brake and rotor spinning at a speed between 4 and 6.4 revolutions per second. A constant voltage has been applied to the brake's coils, and the brake speed time history has been measured by a tachometer and recorded using a data-acquisition system. The brake speed time history has been differentiated numerically and multiplied by the known rotor inertia in order to determine the braking torque. The experiments have been run using a number of different voltage levels and two different rotor inertias. In addition, purely electronic experiments have been performed in order to measure the coil's resistance $R_{b}$ and time constant $\tau_{b}=L_{b} / R_{b}$.

The following model for the magnetorheological brake yields a good fit to the experimental data:

$$
\begin{aligned}
\frac{\mathrm{d} i_{b}}{\mathrm{~d} t} & =-\frac{i_{b}}{\tau_{b}}+\frac{v_{b}}{L_{b}} \\
n_{b}= & \operatorname{sign}\left(\dot{\theta}_{r}\right)\left[\left|i_{b}\right|\left(0.7723-21.3180 i_{b}^{2}-3.4478 i_{b}^{4}\right)\right. \\
& \left.+\left(15.0850 i_{b}^{2}+12.5460 i_{b}^{4}+0.3631 i_{b}^{6}\right)\right]
\end{aligned}
$$

where $i_{b}$ is the current in the brake coils in amps, $v_{b}$ is the control voltage that gets applied across the brake coils, $L_{b}$ is the inductance of the brake coils in henries, $n_{b}$ is the braking torque of the coils in $\mathrm{N}-\mathrm{m}$, and $\dot{\theta}_{r}$ is the rotation rate of the wire-wrapping drum relative to the main spacecraft body in rad/s. The coil's electrical constants are $\tau_{b}=0.030 \mathrm{~s}$ and $L_{b}=0.2475 \mathrm{H}$.

The brake model in Eqs. (5a) and (5b) consists of a simple impedance-induced lag model, Eq. (5a), and a static nonlinear current/torque curve, Eq. (5b). The torque is independent of speed, except that it always acts to oppose motion, as in a Coulomb friction model. The torque/current relationship given in Eq. (5b) is an even function of $i_{b}$ and is valid up to $\left|i_{b}\right|=2.5 \mathrm{~A}$. This function is plotted in Fig. 5. Figure 5 shows that the torque increases almost linearly with current up to about $1 \mathrm{~A}$ of current. Above $1 \mathrm{~A}$, the curve begins to flatten out because the magnetorheological effect approaches its saturation point.

The torque $n_{b}$ enters the wire-boom dynamics model of Ref. 3 as a replacement for the damping term $\left(b_{\mathrm{rb} 1} \dot{\theta}_{r}+b_{\mathrm{rb} 2} \dot{\theta}_{r}\left|\dot{\theta}_{r}\right|\right)$. This damping term appears in Ref. 3 as the third element of the $\boldsymbol{n}_{\mathrm{rb}}$ torque vector in the three-dimensional model-the first two components of $\boldsymbol{n}_{\mathrm{rb}}$ are constraint torques - and in the first two equations of the planar model, Eqs. (10a) and (10b) of Ref. 3. The three-dimensional and

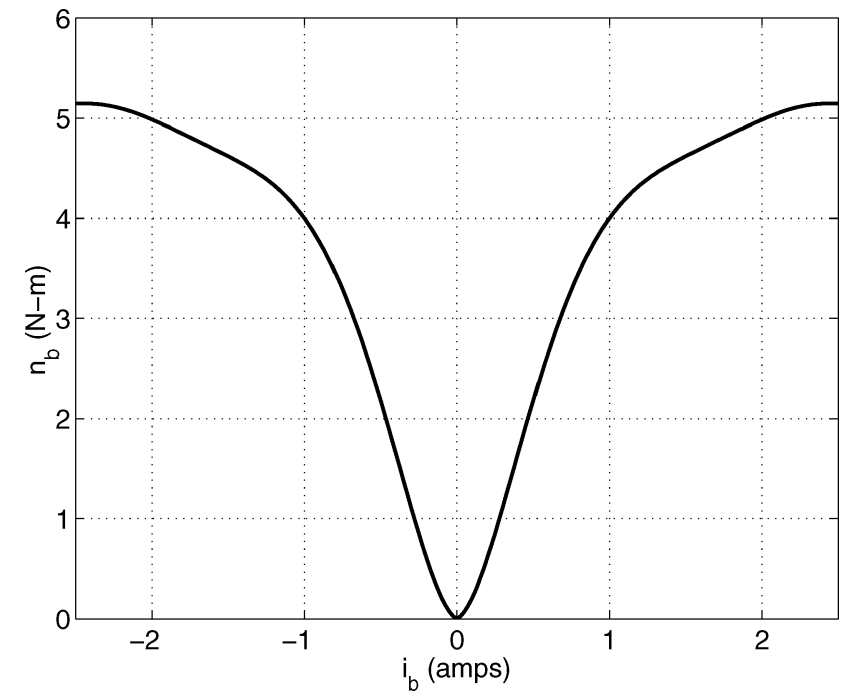

Fig. 5 Torque vs current characteristic for the magnetorheological brake.

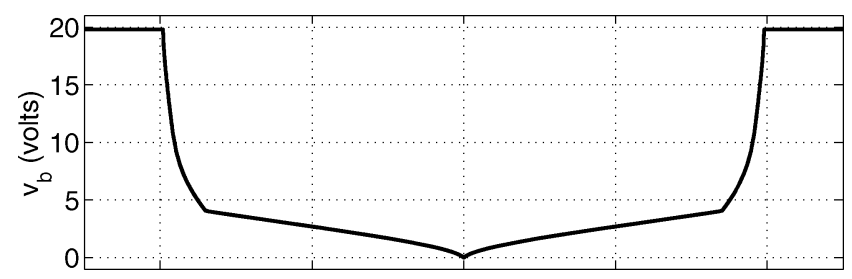

Fig. 6a Damper voltage feedback control law.

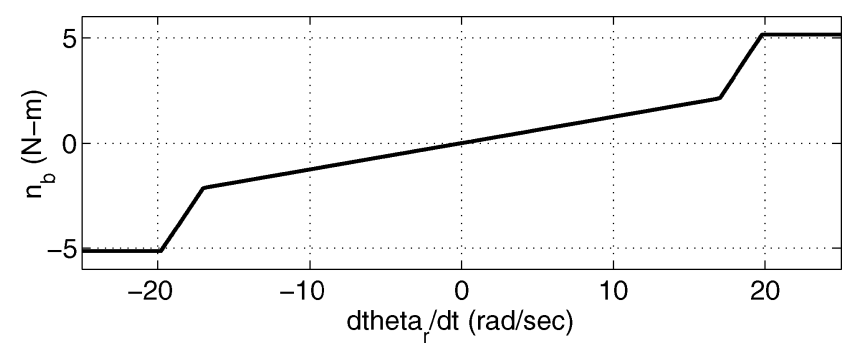

Fig. 6b Corresponding dependence of damper torque on relative rotation rate.

planar simulations of Ref. 3 have been updated for the present study by making these substitutions and by adding the damper current as a new state. Its dynamics are modeled by Eq. (5a). In theory, the brake rotor's moment of inertia about its rotation axis should be added to the drum's spin-axis moment of inertia in the model. In practice, the brake rotor's moment of inertia is negligible compared to that of the drum.

The brake voltage gets controlled via feedback of $\dot{\theta}_{r}$, the drum's rotation rate relative to the main spacecraft body. This feedback signal is derived via differentiation of an angle encoder's output. The feedback control law that has been implemented is shown in Fig. 6a. It has been designed to give the piecewise linear dependence of $n_{b}$ on $\dot{\theta}_{r}$ that is shown in Fig. 6b. This control law has one damping rate for $\left|\dot{\theta}_{r}\right| \leq 17 \mathrm{rad} / \mathrm{s}$, a higher damping rate for $17 \mathrm{rad} / \mathrm{s}<\left|\dot{\theta}_{r}\right| \leq 20$ $\mathrm{rad} / \mathrm{s}$, and a constant damping torque for $20 \mathrm{rad} / \mathrm{s}<\left|\dot{\theta}_{r}\right|$. Figure $6 \mathrm{~b}$ uses the current/voltage relationship $i_{b}=v_{b} / R_{b}$, which presumes that the current dynamics in Eq. (5a) have reached steady state.

The original damper designs of Ref. 3 presumed that the damper could produce torques greater than $5 \mathrm{~N}-\mathrm{m}$, but, as Fig. 5 shows, this is not the case for the magnetorheological brake that has been used for the SIERRA design. This limitation made it difficult to design a damping law that would avoid a rewrapping oscillation during the initial period of wire-boom unwrapping and that would 
be insensitive to possible manufacturing errors. The damping law depicted in Fig. 6 has overcome this difficulty. Its simulated planar and three-dimensional deployment performance satisfactorily avoids the possibility that the wire booms will rewrap or become entangled with each other. As in Ref. 3, the simulation study of this damping system's performance has considered the effects of parameter uncertainty caused by manufacturing variability and the effects of initial condition uncertainty. The details of this study have been omitted for the sake of brevity. The reader can consult Ref. 3 in order to learn more about the simulation methods that have been used to explore the deployment's performance robustness.

\section{Ground Tests of Planar Dynamics of Deployment System}

A number of ground tests of the deployment system were carried out. Most of the tests used only prototype hardware. The last test was the most realistic. It used flight hardware. Its three principal goals were to check the functioning of the mechanism that released both the wire booms and the drum's relative rotation bearing, to ensure that the damper feedback controller operated properly, and to check for agreement between the initial two-dimensional transients of the deployment and simulation-based predictions of this response.

These tests were conducted by mounting the main spacecraft body on a spin table. The spin table constrained the main body to spin about a single axis. This constraint permitted the tests to evaluate the system's principal energy dissipation mechanism, but it precluded evaluation of the system's full three-dimensional transient dynamics. A motor spun the table up to SIERRA's nominal initial spin rate, four revolutions per second. Once at the proper initial speed, the motor shut off and allowed the table to spin freely. The wire-boom's and the drum's relative rotation were then released, and the deployment began. The wire booms began to unwrap, relative motion built up between the drum and the main spacecraft body, the damper damped out this motion, and the whole system slowed down as a result of the increase in total system inertia and because of unwanted external drag effects. Although not supported against gravity, the wire booms did not sag appreciably during the initial part of the deployment because the centrifugal acceleration was much larger than the acceleration of gravity. Towards the end of a test, however, the wire booms sagged as a result of the system's decreased rotation speed, and they hit the floor of the test facility in many cases. The test results ceased to be meaningful when there was too much sag of the booms or if the booms hit the floor of the test facility.

The test that used flight hardware was conducted at the NASA Wallops facility. The spin table was inverted and mounted to the top of a high bay during this test. This configuration allowed the wire booms to execute their natural motions without ever hitting the floor of the facility. The only factor that limited the useful duration of this test was the eventual large boom sag under the influence of gravity.

Various data were collected during each test. Time histories were recorded for the brake voltage $v_{b}$, the drum's relative articulation angle $\theta_{r}$, and the spin table rotation rate $\omega_{z}$. The system was videotaped during each test as a way of recording the location time history of each wire boom.

Figure 7 compares simulation results with data from the ground test that used flight hardware. The top plot presents the damper feedback voltage time histories for these two cases, and the bottom plot presents the two relative spin-rate time histories for the drum. The simulation for this case uses an increased value of $I_{\mathrm{zzb}}$, the spin-axis moment of inertia of the main spacecraft body, in order to account for the added inertia of the spin table. The two plots show agreement between the simulation and the test for the initial and final parts of the deployment, but disagreement during an interim period that extends from $t=0.25$ to $3.5 \mathrm{~s}$. The initial $0.25 \mathrm{~s}$ of agreement correspond to the time when the drum undergoes an initial despin that ramps up its spin rate relative to the main spacecraft body. The period of disagreement includes the majority of the time during which the wire booms unwrap (0-2 s) and most of the period of significant in-plane pendulum motion of the booms after they have completely unwrapped (2-4 s). The experiment exhibits a lower

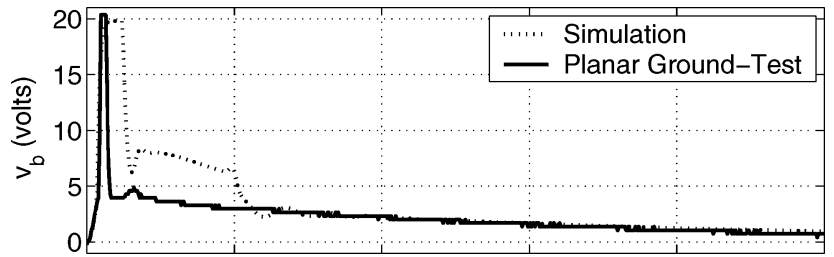

a)

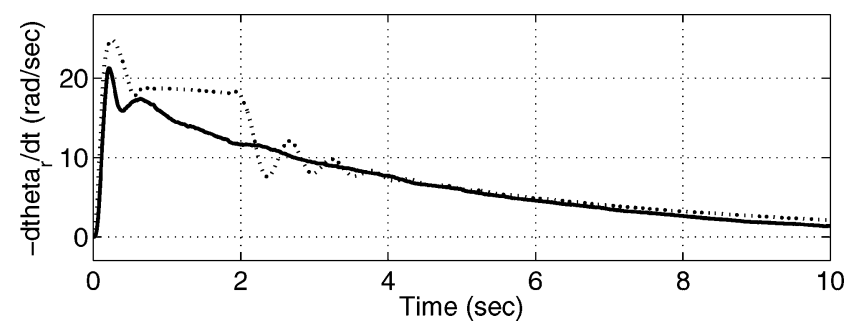

b)

Fig. 7 Simulated and ground-test time histories a) for the damper voltage $v_{b}$ and $b$ ) for the drum's relative rotation rate $-\dot{\theta}_{r}$.

relative rotation speed between the drum and the main body than the simulation for most of the time when the cables are unwrapping. This discrepancy gives rise to the differences between the two damper feedback voltage time histories. Also missing from the experiment is significant collective wire-boom pendulum motion subsequent to unwrapping. This fact shows up on the lower plot as a lack of oscillations of the solid black curve between 2 and $4 \mathrm{~s}$.

The experiment that produced Fig. 7 was deemed a partial success. The release mechanism worked well, and the wire booms deployed successfully until gravity caused their sag to violate the simulation model's assumptions. The initial spike of the relative rotation rate between the main spacecraft body and the wire-wrapping drum agreed fairly well with simulation. The disagreements between simulation and test on Fig. 7 were considered to be the results of additional damping that was part of the experiment but that would not be present during an actual flight. The two main perturbing effects were thought to be atmospheric drag on the wire booms and viscous damping in the spin table's bearing.

\section{Flight Performance of the System}

The new wire-boom system was built and flown on the SIERRA sounding rocket mission, and telemetry data have been used to assess its performance. The sounding rocket was launched from the Poker Flat Research Range, Alaska, on 14 January 2002. The entire flight lasted about $900 \mathrm{~s}$ and reached an apogee altitude of $735.25 \mathrm{~km}$. Further details about this mission can be found in Ref. 10 .

The mission consisted of a mother spacecraft and two daughter spacecraft that separated from the mother. Figure 8 shows the two daughter spacecraft in stowed configuration and part of the mother spacecraft during preflight integration. The three spacecraft drifted apart to form an expanding triangle in the local horizontal plane. Its maximum dimension was on the order of $1.3 \mathrm{~km}$ at the end of the flight. Each daughter spacecraft was equipped with a wire-boom system in order to allow the mission to make distributed electric field measurements during an auroral event. The heavier mother spacecraft carried a traditional semirigid boom system. The first daughter module separated from the mother spacecraft $136 \mathrm{~s}$ after launch, and the second module separated $23 \mathrm{~s}$ later. Both daughter spacecraft began their wire-boom deployments $7 \mathrm{~s}$ after separation. The mother spacecraft's gas jet attitude control system was used to set up the proper initial spin vector for each daughter spacecraft just prior to separation.

The following final design parameters were used for the wire-boom system on each SIERRA daughter spacecraft: $m_{\text {bd }}=$ $47.74 \mathrm{~kg}, m_{\text {tip }}=0.033 \mathrm{~kg}, I_{\mathrm{xxbd}}=1.832 \mathrm{~kg}-\mathrm{m}^{2}, I_{\mathrm{zzb}}=0.811 \mathrm{~kg}-$ $\mathrm{m}^{2}, \quad I_{\mathrm{zzd}}=0.042 \mathrm{~kg}-\mathrm{m}^{2}, I_{\mathrm{zzbd}}=0.853 \mathrm{~kg}-\mathrm{m}^{2}, \quad z_{r}=0.0583 \mathrm{~m}$, $\rho_{r}=0.152 \mathrm{~m}$, and $l_{f}=2.837 \mathrm{~m}$, where $I_{\mathrm{zzbd}}=I_{\mathrm{zzb}}+I_{\mathrm{zzd}}$ because 


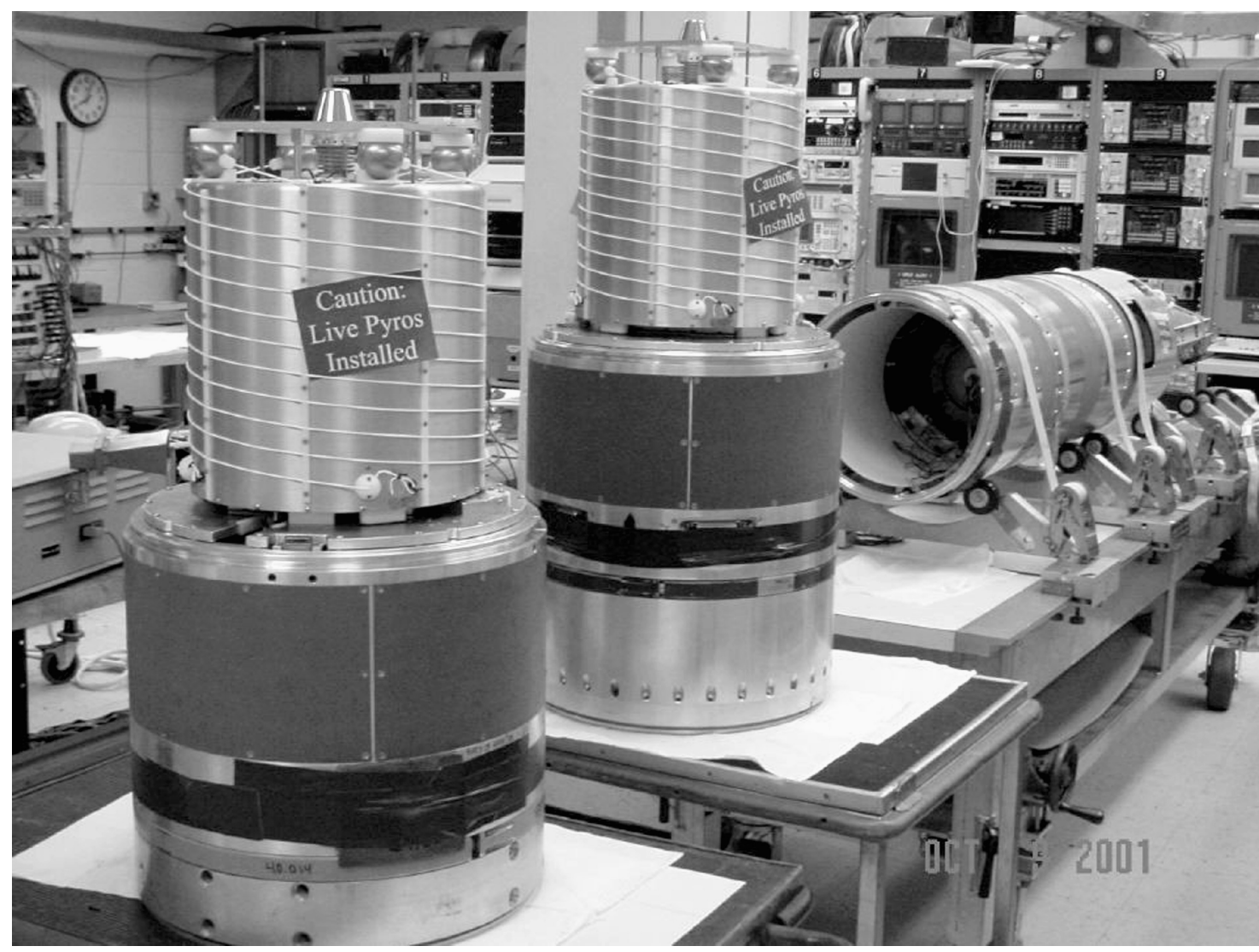

Fig. 8 Two SIERRA daughter spacecraft with wire booms stowed about drums (left and center) and part of SIERRA mother spacecraft (right).

$I_{\text {zzb }}$ is the $z$-axis inertia of the daughter spacecraft's main body and $I_{\mathrm{zzd}}$ is the $z$-axis inertia of the drum. Note that $I_{\mathrm{zzd}}$ is called $I_{\mathrm{zzr}}$ in Ref. 3 because the drum is called a ring in that paper.

The telemetry stream included data from all three spacecraft. Data from a three-axis gimbaled gyroscope and from a horizon crossing indicator were returned from the mother spacecraft. Magnetometer data were recorded for all three spacecraft. Additional telemetry from each daughter payload included encoder data for the angle between the wire-wrapping drum and the spacecraft body along with the voltage that was fed back to the magnetorheological damper.

The telemetry data indicate that the two wire-boom systems functioned as well or better than predicted in simulation. Figure 9 shows the wire-wrapping drum's relative spin-rate time history. This plot is similar to the bottom plot of Fig. 7, except that it compares simulation results with two sets of actual flight results, one for the daughter spacecraft that was mounted aftmost in the launch configuration and the other for the daughter module that was mounted foremost. The articulation rate time histories for the two daughter spacecraft, the black solid curve and the gray dashed curve, are remarkably similar to the simulation's predicted time history, the dotted gray curve. All three curves show a sharp initial spike as the wire booms slow the drum's absolution rotation. Next comes a nearly flat section that lasts for about $2 \mathrm{~s}$ while the wire booms finish unwrapping. After the unwrapping phase, the booms undergo collective in-plane pendulum oscillations at a frequency of about $2 \mathrm{~Hz}$, and these oscillations cause the oscillations of the drum's relative rotation rate that are evident in Fig. 9. These oscillations die out by about $5 \mathrm{~s}$ after the start of the deployment.

The one significant difference between the simulated time history of Fig. 9 and the flight data is that the former time history takes longer to damp the drum articulation rate to zero. This implies that the lowspeed damping torque of the magnetorheological brake is slightly stronger than is indicated by the model in Eq. (5b). This discrepancy between the simulation and the flight data is acceptable because it represents an improvement over the predicted performance; the system reaches steady state more quickly.

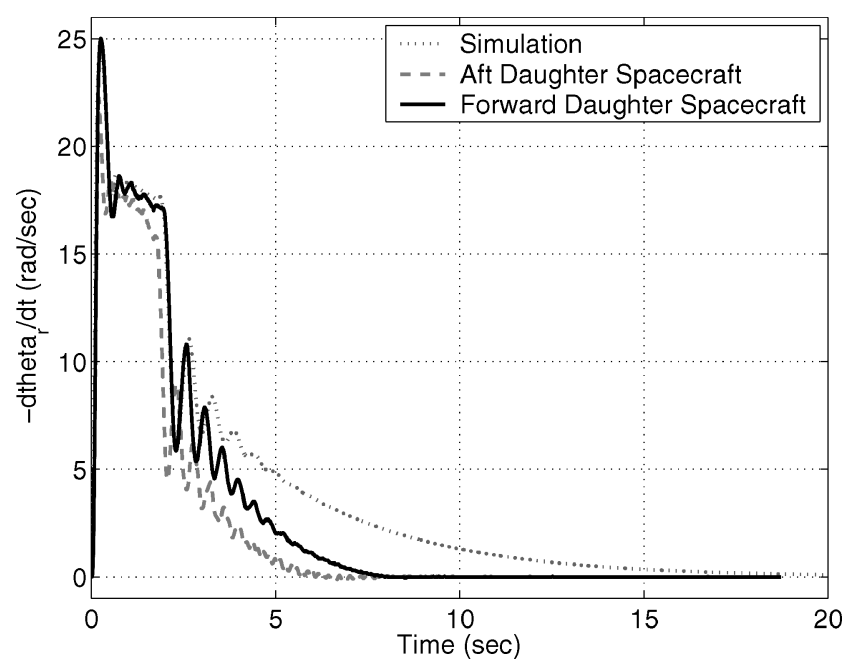

Fig. 9 Simulated and flight telemetry time histories for the relative rotation rate of the wire-wrapping drum $-\dot{\theta}_{r}$.

The attitude-rate time histories of the two SIERRA daughter spacecraft have been estimated by using their telemetered magnetometer data and the estimation techniques of Ref. 11. Reference 11 develops a batch estimator and a Kalman filter, each of which can estimate three-axis attitude rate using only magnetometer data. Neither estimator requires attitude knowledge, and neither needs an Earth magnetic field model. This latter simplification puts a lower bound on the achievable accuracy of about $0.12 \mathrm{deg} / \mathrm{s}$. Both estimators use the kinematics of the magnetic field direction vector as observed in spacecraft coordinates, and both use Euler's equation for a rigid spacecraft.

One can apply the rigid-body estimators of Ref. 11 to the flexible, wire-boom-equipped SIERRA daughter spacecraft if one does 
so with care. A rigid-body attitude model can be applied after the deployment transients have died out because the dominant mode of motion during this flight phase is a slightly unstable nutation oscillation. The wire booms undergo slow out-of-plane pendulum oscillations in this nutation mode. The angle $\phi_{i}$ of Fig. 1 oscillates at the nutation frequency under the influence of the time-varying centrifugal force that is generated by the time-varying spin vector direction. The spin vector moves with respect to spacecraft coordinates in much the same way that it would nutate for a rigid minor axis spinner. In the limit of small nutations, there is no difference between the SIERRA spin vector nutations and those of a minor axis spinner with properly adjusted inertia ratios. This fact allows the estimation techniques of Ref. 11 to be used to estimate the steadystate SIERRA spin-vector time history if its nutation amplitude is not too large and if the correct equivalent moment of inertia matrix is used. The Kalman filter of Ref. 11 includes the option to estimate corrections to five of the six inertia matrix elements of its rigidbody model; so, this estimator is well suited for use on the SIERRA daughter spacecraft because it can make the necessary inertia matrix adjustments.

The pre- and postdeployment spin-vector time histories for the two SIERRA daughter spacecraft have been estimated, and the consistency of the rigid-body estimation model has been validated in two ways. First, the variations of the estimated spin-rate direction in spacecraft coordinates have been checked for smallness. The estimated spin-rate direction time history of the aft daughter spacecraft stays within 9 deg of the nominal spin axis for the duration of the flight, and the forward daughter spacecraft maintains its spin-vector direction within $5.5 \mathrm{deg}$ of the nominal spin axis. These angles are small enough to be consistent with the assumptions of the rigid-body nutation model. A further test has used the estimated spin-vector time histories to calculate estimated pseudo-angular-momentumvector time histories. These time histories use the rigid-body assumption and the estimator's adjusted moment-of-inertia matrices. The resulting pseudo-angular momentum time histories, although not meaningful in a physical sense, have nearly constant vector magnitudes, as they should if the rigid-body approximation is valid. This fact confirms the mathematical reasonableness of the approximation. A similar validation calculates the projections of the estimated pseudo-angular momentum vectors onto the corresponding measured magnetic field directions. These projections are calculated in spacecraft coordinates. They produce very slow time variations that are mathematically consistent with the expected slow inertial variations of the magnetic field direction and the expected constancy of the inertial direction of the pseudo-angular momentum vector. Thus, an adjusted rigid-body model yields a reasonable approximation of the dominant steady-state nutational motion of the wire-boom system and can be used to estimate the system's attitude rates.

The estimated spin-vector time histories can be used to evaluate the performance of the wire-boom system. The first interesting feature of these estimates concerns the net change in the spin rate during the deployment. The preflight simulation predicted that the spin rate after deployment would be 0.419 times the initial spin rate. The aft daughter spacecraft's postdeployment spin rate was 0.422 times its initial spin rate, and the forward daughter's spin rate decreased by a factor of 0.423 during the deployment. These decay factors are all within $1 \%$ of each other, which is a remarkable level of agreement between prediction and flight performance. This close agreement is the result of two factors: tight control of manufacturing tolerances during the fabrication of the wire-boom systems and accurate measurements of the flight units' moments of inertia using a spin balance table.

The postdeployment estimates of the spin vector time histories can be used to estimate the coning half-angle time history for each daughter spacecraft. The coning half-angle is the angle between the estimated pseudo-angular momentum vector and the nominal spacecraft spin axis. The estimated coning half-angle time histories for the two daughter payloads are plotted in Fig. 10. The exponential growth of these two curves shows that each spacecraft's nutation mode was unstable, but not unstable enough to violate the mission's pointing requirement. This requirement called for the coning half-

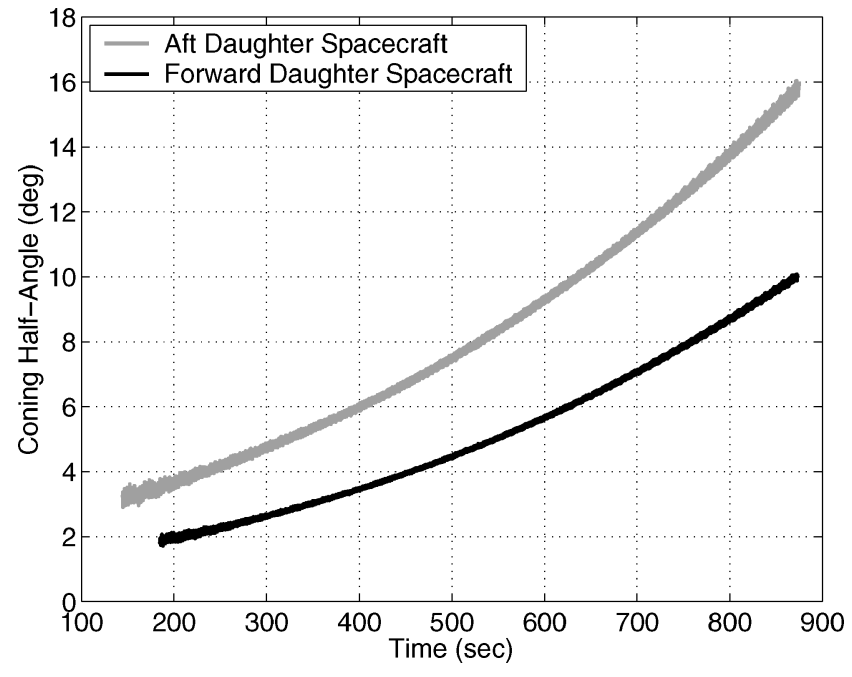

Fig. 10 Estimated coning half-angle time histories for the two SIERRA daughter spacecraft after wire-boom deployment.

angle to remain below $45 \mathrm{deg}$ for the duration of the flight. The performance shown in Fig. 10 surpasses this requirement by a factor of three or more.

The rate of exponential nutation growth experienced in the SIERRA flight is roughly equivalent to the rate predicted by the pendulum tests and the simulation. The Eq. (4) damping model causes the coning half-angle in Fig. 3 to grow from 4 to 20 deg in $700 \mathrm{~s}$. In actual flight, the forward daughter spacecraft experienced a rate of growth that was $5 \%$ larger than the simulation. Its estimated coning half-angle in Fig. 10 grew from 1.9 to 10.0 deg in 689 s. The aft daughter spacecraft experienced a rate of coning growth that was $5 \%$ lower than the simulation. Its coning half-angle grew from 3.2 to $15.9 \mathrm{deg}$ in $732 \mathrm{~s}$. Thus, the simulation has proved remarkably accurate in its ability to predict the rate of coning growth.

In summary, the first flight test of the yo-yo wire-boom deployment system has been a success. The deployment transients and the postdeployment steady-state performance met or exceeded expectations. The wire-boom's deployment transients died out in less than $10 \mathrm{~s}$ and displayed no evidence of dangerous trends that might lead to tangling of the booms. The system maintained a low level of coning during the deployment so that the coning half-angle immediately after deployment was less than $3.5 \mathrm{deg}$. Subsequently, the coning half-angle grew as the result of the unstable nutation mode, but this growth occurred at a rate that was about equal to experimentally based projections, and this angle was no more than 16 deg by the end of the flight.

\section{Conclusions}

A practical implementation of a new yo-yo-type wire-boom deployment system has been developed and flown on a sounding rocket mission. This system allows wire booms to be deployed from a spinning spacecraft in a matter of seconds. One critical element of a practical system is a semi-active magnetorheological brake, which provides the energy dissipation that is needed in order to prevent rewrapping of the booms after they have reached their full extensions. Another key development has been an ability to design a system that has significant axial offsets between the spacecraft center of mass, the boom base attachment points, and the stowed positions of the boom tip masses. Such offsets allow one to design a relatively simple deployment mechanism, but they force the system to tolerate a slightly unstable nutation mode. This slight instability can be restricted to manageable levels for a sounding rocket mission through the use of wire booms that exhibit low damping of their pendulum motion.

A system with four 3-m booms has been built and flown. Ground tests and flight telemetry data have demonstrated that the system meets or exceeds simulation-based predictions of its performance. The booms deployed in less than $10 \mathrm{~s}$, and the coning half-angle of 
the deployed system's unstable nutation mode stayed below 16 deg for the 700-s postdeployment portion of the mission.

Although 3-m booms have been used on this first mission, simulations and analyses predict that longer systems are practical. The hardware design can be scaled to use longer wire booms by wrapping them around a drum more times in the stowed configuration. The deployment time increases, but most of the analysis and design principles used for the 3-m system apply equally for longer systems. One exception is that the wire-boom helical wrapping pattern on the drum must be modified to be nonuniform in order to avoid excessive wobble during transients. It must start out steep during the initial unwrapping phase and then flatten out. One new mission plans to use 6-m booms wrapped around the same basic drum/brake design.

\section{Appendix: Derivation of Nutational Stability Criterion}

The stability of the nominal spin axis can be determined by analyzing the dependence on spin direction of the quasi-static moment of inertia. Suppose that the instantaneous spin unit direction vector is $\hat{\omega}=[0 ;-\sin \eta ; \cos \eta]$, that is, suppose that the instantaneous spin direction is tilted away from the $+z$ axis toward the $-y$ axis by the angle $\eta$. Suppose, also, that the booms are in their quasisteady orientations, which are aligned with the centrifugal force vector as measured at each boom root. Then the spin-axis moment of inertia of the whole system measured about its center of mass is

$$
\begin{aligned}
& I_{\text {spin }}(\eta)=I_{\mathrm{xxbd}} \sin ^{2} \eta+I_{\mathrm{zzbd}} \cos ^{2} \eta+m_{\mathrm{bd}}\left(l_{a} \sin \alpha+z_{a} \sin \eta\right)^{2} \\
& \quad+2 m_{\mathrm{tip}}\left\{\left(\rho_{r}+l_{f} \cos \alpha\right)^{2}+\left[\left(z_{r}-z_{a}\right) \sin \eta+\left(l_{f}-l_{a}\right) \sin \alpha\right]^{2}\right. \\
& \left.\quad+\left(\rho_{r} \cos \eta+l_{f}\right)^{2}+\left[\left(z_{r}-z_{a}\right) \sin \eta-l_{a} \sin \alpha\right]^{2}\right\}
\end{aligned}
$$

where $l_{a}=2 l_{f} m_{\text {tip }} /\left(m_{\text {bd }}+4 m_{\text {tip }}\right)$ and $z_{a}=4 z_{r} m_{\text {tip }} /\left(m_{\text {bd }}+4 m_{\text {tip }}\right)$.

The centrifugal forces and the axial offset $z_{r}$ combine to cant the $+x$ and $-x$ wire booms toward the $+y$ axis by the angle $\alpha$ when the spin tilt angle $\eta$ is nonzero. The cant angle $\alpha$ is defined as an implicit function of the spin axis tilt angle $\eta$ via the following equation:

$$
0=\left(z_{r}-z_{a}\right) \sin \eta \cos \alpha-l_{a} \sin \alpha \cos \alpha-\rho_{r} \sin \alpha
$$

which constitutes the quasi-static equilibrium condition for the $+x$ and $-x$ wire booms.

Spin about the $z$ axis of this system will be locally stable if $I_{\text {spin }}(\eta)$ has a local maximum at $\eta=0$. This is a minimum-energy condition. This condition will be met if the first derivative of $I_{\text {spin }}(\eta)$ with respect to $\eta$ is zero and if the second derivative is negative. These derivatives can be evaluated by differentiating Eq. (A1). As part of this process, one must differentiate Eq. (A2) in order to evaluate the derivatives of $\alpha(\eta)$. The results of this calculation show that $\left.\left(\mathrm{d} I_{\text {spin }} / \mathrm{d} \eta\right)\right|_{0}=0$ and that

$$
\begin{aligned}
& \left.\frac{\mathrm{d}^{2} I_{\text {spin }}}{\mathrm{d} \eta^{2}}\right|_{0}=2\left\{I_{\text {xxbd }}-I_{\text {zzbd }}-2 m_{\text {tip }} \rho_{r}\left(\rho_{r}+l_{f}\right)\right. \\
& \left.+z_{r}^{2}\left[\frac{4 m_{\text {tip }}\left(1+l_{f} / 2 \rho_{r}\right) m_{\text {bd }}}{4 m_{\text {tip }}\left(1+l_{f} / 2 \rho_{r}\right)+m_{\text {bd }}}\right]\right\}
\end{aligned}
$$

Thus, the stability condition in Eq. (1) is simply the requirement that this second derivative be negative.

The validity of the preceding analysis is dependent on the symmetry of the system. For a general system one must consider $I_{\text {spin }}\left(\eta_{x}, \eta_{y}\right)$, which is a function of two orthogonal rotations of the spin axis away from the $+z$ axis, one about the $x$ axis and the other about the $y$ axis. The function $I_{\text {spin }}\left(\eta_{x}, \eta_{y}\right)$ must be a local maximum at $\left(\eta_{x}, \eta_{y}\right)=(0,0)$ in order for the system to be stable. This is true if the matrix $\boldsymbol{H}=\left[\mathrm{d}^{2} I_{\text {spin }} / \mathrm{d} \eta_{x}^{2}, \mathrm{~d}^{2} I_{\text {spin }} / \mathrm{d} \eta_{x} \mathrm{~d} \eta_{y}\right.$; $\left.\mathrm{d}^{2} I_{\text {spin }} / \mathrm{d} \eta_{x} \mathrm{~d} \eta_{y}, \mathrm{~d}^{2} I_{\text {spin }} / \mathrm{d} \eta_{y}^{2}\right]$ is negative definite.

The symmetry of the system implies that

$$
\begin{aligned}
& {[\cos \beta, \sin \beta] \boldsymbol{H}\left[\begin{array}{c}
\cos \beta \\
\sin \beta
\end{array}\right]} \\
& \quad=[\cos (\beta+\pi / 2), \sin (\beta+\pi / 2)] \boldsymbol{H}\left[\begin{array}{c}
\cos (\beta+\pi / 2) \\
\sin (\beta+\pi / 2)
\end{array}\right]
\end{aligned}
$$

for any angle $\beta$. Equation (A4) expresses the fact that the quasistatic spin inertia does not change if one rotates the spin vector $\pi / 2 \mathrm{rad}$ about the $z$ axis. This equation is valid because the system consists of an axially symmetric main-body/drum to which four wire booms are attached at equally spaced intervals. If one evaluates Eq. (A4) at the angle $\beta=0$ and at the angle $\beta=\pi / 4$, then the two resulting equations can be used to show that $\boldsymbol{H}$ equals the identity matrix times the second derivative given in Eq. (A3). Therefore, $\boldsymbol{H}$ is negative definite whenever the stability criterion given in Eq. (1) is satisfied.

The local validity of the Eq. (1) stability criterion has been checked by using the linearized steady-state dynamics model of Ref. 3. If wire-boom damping is included in the model, then the system's nutation-mode characteristic values cross from stable to unstable at the parameter values that cause Eq. (1) to be an exact equality.

Although the stability criterion in Eq. (1) has been proven correct only in a local sense, it is conjectured to define a global minimumenergy condition. Reference 3's restricted criterion has been proven to be a global minimum-energy criterion, and its similarity to Eq. (1) leads one to suspect that Eq. (1) also represents a global minimumenergy criterion.

The nutation stability criterion does not depend on the spin frequency. This is in contrast to a stability study in Ref. 12 for a flexible major-axis spinning spacecraft with a tether. The present system's stability does not depend on spin rate because its wire booms are assumed to have zero bending stiffness. The loss of stability above a given frequency reported for the system in Ref. 12 comes about because centrifugal force terms overwhelm boom bending stiffness.

\section{Acknowledgments}

This work was supported in part by NASA Grant NAG5-5233, Sounding of the Ion Energization Region: Resolving Ambiguities. Former students Paul Otañez, Lael Fisher, Toni Poloso, and Kirana Ramakrishna developed and tested early prototype systems and performed the bench tests that were used to characterize the magnetorheological brake.

\section{References}

${ }^{1}$ Longman, R. W., and Fedor, J. V., "Dynamics of Flexible Spinning Satellites with Radial Wire Antennas," Acta Astronautica, Vol. 3, Nos. 1 and 2, 1976, pp. 17-37.

${ }^{2}$ Hubert, C., "The Attitude Dynamics of Dynamics Explorer A," AIAA Paper 81-123, Aug. 1981.

${ }^{3}$ Psiaki, M. L., Kinter, P. M., Jr., and Powell, S. P., "Rapid Energy Dissipation in a Yo-Yo-Type Wire Boom Deployment System,' Journal of Guidance, Control, and Dynamics, Vol. 23, No. 3, 2000, pp. 483-490.

${ }^{4}$ Wiesel, W. E., Spaceflight Dynamics, McGraw-Hill, New York, 1989, pp. 141-145.

${ }^{5}$ Schiring, E. E., Heffel, J. W., and Litz, C. J., "Simulation Modeling and Test of a Satellite Despin System,” AIAA Paper 89-3267, Aug. 1989.

${ }^{6}$ McIntyre, J. E., and Miyagi, M. I., "A General Stability Principle for Spinning Flexible Bodies with Application to the Propellant MigrationWobble Amplification Effect," Proceedings of the Symposium on Dynamics and Control of Non-Rigid Spacecraft, ESA, Noordwijk, The Netherlands, 1976, pp. 159-175.

${ }^{7}$ Psiaki, M. L., Klatt, E. M., Kintner, P. M., Jr., and Powell, S. P., "Attitude Estimation for a Flexible Spacecraft in an Unstable Spin," Journal of 
Guidance, Control, and Dynamics, Vol. 25, No. 1, 2002, pp. 88-95.

${ }^{8}$ Avallone, E. A., and Baumeister, T., III (eds.), Marks' Standard Handbook for Mechanical Engineers, 9th ed., McGraw-Hill, New York, 1987, p. 5-69.

${ }^{9}$ Carlson, J. D., Catanzarite, D. M., and St. Clair, K. A., "Commercial Magneto-Rheological Fluid Devices," Proceedings of the 5th International Conference on Electro-Rheological Fluids, Magneto-Rheological Suspensions and Associated Technology, edited by W. A. Bullough, World Scientific, Singapore, 1996, pp. 20-28.

${ }^{10}$ Powell, S. P., Klatt, E. M., and Kintner, P. M., Jr., "Plasma Wave In- terferometry Using GPS Positioning and Timing on a Formation of Three Sub-Orbital Payloads," Proceedings of the ION GPS Conference, Inst. of Navigation, Fairfax, VA, 2002, pp. 145-154.

${ }^{11}$ Psiaki, M. L., and Oshman, Y., "Spacecraft Attitude Rate Estimation from Geomagnetic Field Measurements," Journal of Guidance, Control, and Dynamics, Vol. 26, No. 2, 2003, pp. 244-252.

${ }^{12}$ Vigneron, F. R., Jablonski, A. M., Chandrashaker, R., and Tyc, G., "Damped Gyroscopic Modes of Spinning Tethered Space Vehicles with Flexible Booms," Journal of Spacecraft and Rockets, Vol. 34, No. 5, 1997, pp. 662-669. 